\title{
Thyroid Malignancy and Suicide Risk: An Analysis of Epidemiologic and Clinical Factors
}

\author{
${ }^{1}$ Nathaniel J Walsh, ${ }^{2}$ Asif M Talukder, ${ }^{3}$ Andrew G Lawson, ${ }^{4}$ Amel X Komic, ${ }^{5}$ Brian P Bateson \\ ${ }^{6}$ Andrew J Jones, ${ }^{7}$ Edward J Kruse
}

\begin{abstract}
Aim: To date, there has been no specific examination of suicide rates and factors associated with suicide in thyroid cancer. The aim of this study is to examine suicide incidence and associated factors in thyroid cancer patients from 1973 to 2013.
\end{abstract}

Materials and methods: The Surveillance, Epidemiology, and End Results (SEER) database of the National Cancer Institute was queried to identify patients with thyroid cancer from 1973 to 2013. Comparison data with the general US population were derived from the Centers for Disease Control and Prevention's (CDC) National Center for Injury Prevention and Control using the Web-based Injury Statistics Query and Reporting System (WISQARS). Standardized mortality ratios (SMRs) and multivariable logistic regression models generated odds ratios (ORs) for the identification of factors associated with suicide.

Results: Overall, 154 suicides among 168,339 patients were identified. There was no statistically significant difference in suicide rate with respect to age, marital status, median household income, surgical intervention, stage at diagnosis, or histologic subtype. On multivariable analysis male gender and Caucasian race were associated with increased risk of suicide with ORs of 5.39 and 3.17 respectively.

Conclusion: Race and gender appear to influence suicide rates in patients with thyroid cancer. Females and whites were more likely to commit suicide as noted previously. There was no statistically significant relationship between suicide and marital status, income, mode of radiation therapy, and the role of surgical intervention. These results, coupled with further studies and analyses, could be used to formulate a comprehensive suicide risk factor scoring system for screening all cancer patients.

Clinical significance: Thyroid cancer is very treatable with excellent long-term survival and outcomes. Improved screening and risk factor stratification in these patients could decrease the incidence of suicide in thyroid cancer patients.

Keywords: Mortality, Standardized mortality ratio, Suicide, Surveillance epidemiology and end results database, Thyroid cancer.

\footnotetext{
${ }^{1-5}$ Resident, ${ }^{6}$ Student, ${ }^{7}$ Associate Professor

${ }^{1-7}$ Department of Surgery, Medical College of Georgia, Augusta University, Augusta, Georgia, USA

Corresponding Author: Nathaniel J Walsh, Resident Department of Surgery, Medical College of Georgia, Augusta University, Augusta, Georgia, USA, e-mail: nwalsh@augusta.edu
}

How to cite this article: Walsh NJ, Talukder AM, Lawson AG, Komic AX, Bateson BP, Jones AJ, Kruse EJ. Thyroid Malignancy and Suicide Risk: An Analysis of Epidemiologic and Clinical Factors. World J Endoc Surg 2018;10(2):99-102.

\section{Source of support: Nil}

Conflict of interest: None

\section{INTRODUCTION}

The incidence of thyroid cancer is one of the fastest growing in recent years. According to the National Cancer Institute, the incidence has more than doubled since the 1970s. Some postulate the increase could be secondary to identification of a large reservoir of subclinical papillary lesions that may never affect patient health (overdiagnosis). ${ }^{1}$ However, not all epidemiological and clinical data support this hypothesis, and there appears to be a true increase. ${ }^{1}$ Given the surge in incidence, numerous studies have examined socioeconomic factors, psychosocial stressors, disparities, and quality of life associated with thyroid cancer and its survivors. To date, no study has examined suicide risk and incidence associated with the disease.

Increased suicidal tendencies among cancer patients have been well documented. Some studies have demonstrated patients with cancer have nearly twice the incidence of suicide when compared with the general population. ${ }^{2}$ This is particularly interesting given that previous studies have shown that suicide is strongly associated with cancer, but not other disabling and potentially fatal conditions, such as heart attack, hip fracture, or stroke. ${ }^{3}$ Early prevention of suicide in cancer patients becomes even more of a priority given that $36 \%$ of patients will commit suicide within 1 year of diagnosis, and one-third of these patients will do so within the first month. ${ }^{4}$ Given the aforementioned risk for suicide in cancer patients, the National Comprehensive Cancer Network (NCCN) developed the Distress Management Guidelines to provide a means for the rapid assessment of patients for emotional distress and depression in the office. This screening tool is required for all NCCN member cancer centers.

There has been no specific examination of suicide rates and factors associated with suicide in thyroid cancer. The aim of this study is to examine suicide incidence and associated factors in thyroid cancer patients 
from 1973 to 2013 using the SEER database. The literature has demonstrated a perceived lack of support and high levels of distress among thyroid cancer survivors, especially those diagnosed at a younger age. ${ }^{5,6}$ This combined with the fact that most thyroid cancers are very treatable suggests the importance of identifying psychosocial stressors and risk factors for suicide among thyroid cancer patients.

\section{MATERIALS AND METHODS}

The SEER database of the National Cancer Institute was queried to identify patients with all types of thyroid cancer. The SEER database reports data on incidence, prevalence, and survival within registries comprising specific geographic areas representing 30\% of the US population, and the population covered by SEER is comparable to the general US population with regard to measures of poverty and education. The study cohort consists of patients from all 18 registries comprising the SEER database. Mortality and demographic data from 1973 to 2013 from the SEER database were included. Comparison data with the general US population were derived from the CDC's and National Center for Injury Prevention and Control using WISQARS.

Demographic variables of interest included sex, age at diagnosis (by decade), race, income, and marital status. Clinical and pathologic variables included stage at diagnosis, primary site, receipt of surgical therapy, receipt of radiation therapy, and tumor histopathology. The primary outcome was suicide as the cause of death. Standardized mortality ratios and their 95\% confidence intervals (95\% CIs) were calculated, and multivariable logistic regression models generated ORs for the identification of factors associated with suicide in thyroid malignancy. Statistical analysis was done using statistical analysis software (SAS,
Cary, NC) and figures were prepared with GraphPad Prism 6 (San Diego, California).

\section{RESULTS}

Overall, 154 suicides among thyroid cancer patients were identified. This represents $0.7 \%$ of 23,356 deaths in thyroid cancer patients in the SEER data set. In addition to suicide, the other major causes of death are also broken down in Graph 1. Of the patients committing suicide, almost half, 72 (47\%), were over 80 years old. There was no statistically significant difference in suicide rate with respect to age, marital status, median household income, surgical intervention, or histologic subtype. Of those committing suicide, $64 \%$ were male and $94 \%$ were white. The odds of males committing suicide were 5.4 times that of females (95\% CI: 3.8-7.5). The odds of whites committing suicide were 3.2 times that of non-whites (95\% CI: 1.7-6.0). Table 1 summarizes SMRs for the most consistently reliable demographic data.

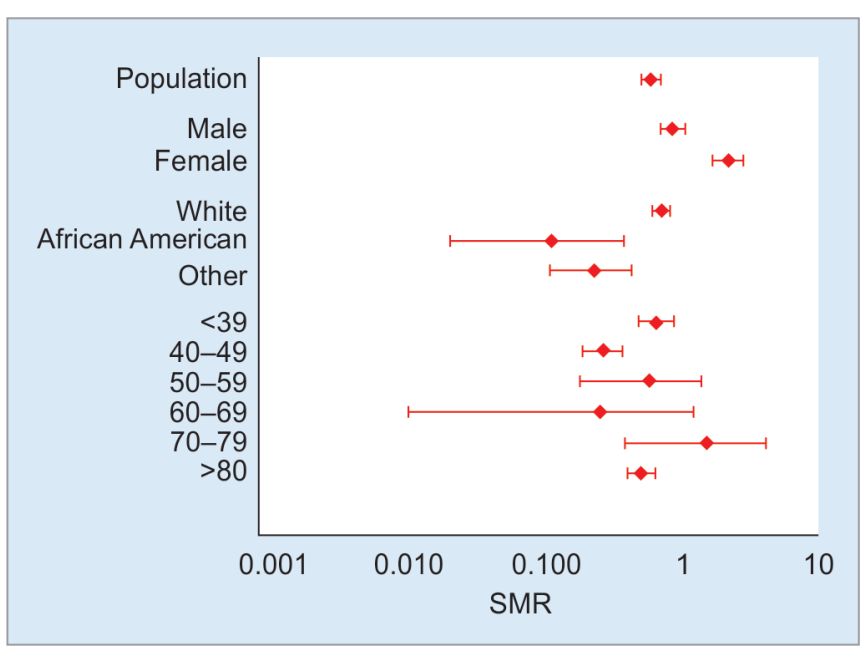

Graph 1: Forest plot of SMR in thyroid cancer patients

Table 1: Incidence of suicide among patients with thyroid cancer by demographic characteristics

\begin{tabular}{|c|c|c|c|c|c|}
\hline Demographics & $\begin{array}{l}\text { Number of } \\
\text { suicides }\end{array}$ & Person-years & $\begin{array}{l}\text { Suicide rate per } 100,000 \\
\text { person-years }\end{array}$ & SMR & $95 \% \mathrm{Cl}$ \\
\hline Thyroid & 154 & 1367749 & 11.00 & 0.59 & $0.50-0.69$ \\
\hline \multicolumn{6}{|l|}{ Sex } \\
\hline Male & 98 & 313001 & 0.22 & 0.85 & $0.69-1.03$ \\
\hline Female & 56 & 1054748 & 0.04 & 2.17 & $1.64-2.78$ \\
\hline \multicolumn{6}{|l|}{ Age (years) } \\
\hline$\leq 39$ & 44 & 523367 & 0.058 & 0.65 & $0.48-0.86$ \\
\hline $40-49$ & 30 & 323265 & 0.065 & 0.27 & $0.19-0.38$ \\
\hline $50-59$ & 4 & 29817 & 0.093 & 0.57 & $0.18-1.38$ \\
\hline $60-69$ & 1 & 19410 & 0.036 & 0.25 & $0.01-1.23$ \\
\hline $70-79$ & 3 & 11227 & 0.186 & 1.50 & $0.38-4.08$ \\
\hline$\geq 80$ & 72 & 460663 & 0.109 & 0.50 & $0.40-0.63$ \\
\hline \multicolumn{6}{|l|}{ Race } \\
\hline African-American & 2 & 76491 & 0.018 & 0.11 & $0.02-0.37$ \\
\hline White & 144 & 1136060 & 0.088 & 0.71 & $0.60-0.83$ \\
\hline Other & 8 & 144621 & 0.038 & 0.23 & $0.11-0.43$ \\
\hline Unknown & 1 & - & - & - & - \\
\hline
\end{tabular}




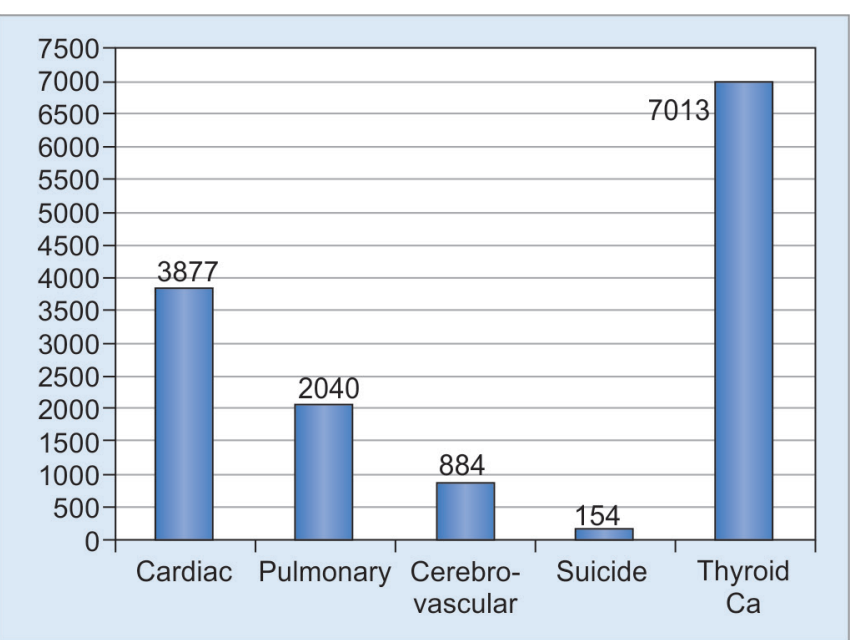

Graph 2: Major causes of death in thyroid cancer

Stage at diagnosis did not have a statistically significant relationship to suicide, although there was a higher frequency of suicides among patients with Stage IV disease. As seen in Graph 2, SMRs for patients with thyroid cancer were 2.17 for females $(95 \% \mathrm{CI}, 1.64-2.78)$, 0.85 for males (95\% CI, 0.69-1.03), 0.11 for AfricanAmericans (95\% CI, 0.02-0.37), 0.71 for whites (95\% CI, $0.60-0.83)$, and 0.23 for other races (95\% CI, 0.11-0.43). On multivariable analysis, male gender and Caucasian race were associated with increased risk of suicide with ORs of 5.39 (95\% CI, 3.89-7.49) and 3.17 (95\% CI, 1.67-6.02) respectively.

\section{DISCUSSION}

To our knowledge, this is the first study to examine the relationship between suicide and thyroid cancer. Suicide has been demonstrated to be increased in the general cancer population as mentioned earlier. Furthermore, suicide has been shown to be a cause of early death in the cancer patient population. Additionally, the incidence of thyroid cancer, especially well-differentiated (papillary, follicular, Hurthle cell), is increasing faster than any other cancer. ${ }^{4}$ Multiple studies have demonstrated the financial and psychosocial ramifications of cancer in patients along with detrimental effects on quality of life. ${ }^{5-8}$ Perhaps even more alarming, financial insolvency related to cancer diagnosis, treatment, and surveillance has been shown to be a risk factor for early mortality in cancer patients. ${ }^{9}$

A couple of interesting trends were noted upon completion of our study. First, females with thyroid cancer were almost twice as likely to commit suicide as females in the general population, SMR 2.17 (95\% CI: 1.64-2.78). Secondly, the odds of whites committing suicide were 3.2 times that of non-whites (95\% CI: 1.7-6.0). Surprisingly, higher stage at diagnosis did not correlate with increase suicide risk. Although previous studies demonstrated worse outcomes in black patients and those of low socioeconomic status with thyroid cancer, our data failed to reveal those as statistically significant risk factors for suicide among thyroid cancer patients. ${ }^{10}$

While our study is limited in its retrospective nature, no other studies, especially with this number of thyroid cancer patients, have examined suicide in this subset of patients. Unfortunately, we were not able to examine the concomitant incidence of preexisting psychiatric disease and thyroid disease in these patients. Given the large control sample from the CDC-WISQARS database, however, we feel any small degree of inherent bias regarding certain preexisting risk factors can be reasonably assumed to be negligible.

\section{CONCLUSION}

To summarize, race and gender appear to influence suicide rates in patients with thyroid cancer. Females and whites were more likely to commit suicide as noted previously. There was no statistically significant relationship between suicide and marital status, income, mode of radiation therapy, and the role of surgical intervention.

Certainly, the incidence of suicide in thyroid cancer patients from the SEER database over the 40-year time was small ( $0.7 \%$ of deaths). Given how treatable thyroid cancer is, particularly the well-differentiated subtypes, we feel these deaths from suicide may represent preventable deaths. With increased awareness, better support infrastructure, and improved emphasis on treating the thyroid cancer patient in a multidisciplinary fashion, suicide rates in thyroid cancer patients, and perhaps all cancer patients, could almost certainly be reduced. This is particularly imperative given the fact that the rate of early suicide after diagnosis of cancer is high, while the 5-year survival in all patients with thyroid cancer is excellent. The American Cancer Society reports 5 -year survival for all stages is $98 \%$.

Our study is an important step in identification of evidence-based risk factors associated with suicide among patients with thyroid cancer. Furthermore, this study emphasizes how critical it is to continue improving screening strategies and management algorithms for patients with risk certain factors and psychosocial stressors. These results, coupled with further studies and analyses, could be used to formulate a comprehensive suicide risk factor scoring system for screening all cancer patients. The new evidence-based risk factor scoring model could subsequently improve on, or be incorporated into, the NCCN Distress Management Guidelines.

\section{CLINICAL SIGNIFICANCE}

The incidence of thyroid cancer has increased significantly in recent years. Most thyroid cancers are very treat- 
able with excellent long-term survival and outcomes. As such, suicide in thyroid cancer patients is a devastating outcome. Improved screening and risk factor stratification in these patients could decrease the overall incidence of suicide in thyroid cancer patients.

\section{REFERENCES}

1. Vigneri R, Malandrino P, Vigneri P. The changing epidemiology of thyroid cancer: why is incidence increasing? Curr Opin Oncol 2015 Jan;27(1):1-7.

2. Misono S, Weiss NS, Fann JR, Redman M, Yueh B. Incidence of suicide in persons with cancer. J Clin Oncol 2008 Oct;26(29):4731-4738.

3. Cole TB, Bowling JM, Patetta MJ, Blazer DG. Risk factors for suicide among older adults with cancer. Aging Ment Health 2014 Sep;18(7):854-860.

4. Johnson TV, Garlow SJ, Brawley OW, Master VA. Peak window of suicides occurs within the first month of diagnosis: implications for clinical oncology. Psychooncology 2012 Apr;21(4):351-356.

5. Easley J, Miedema B, Robinson L. It's the "good" cancer, so who cares? Perceived lack of support among young thyroid cancer survivors. Oncol Nurs Forum 2013 Nov;40(6): 596-600.

6. Roerink SH, de Ridder M, Prins J, Huijbers A, de Wilt HJ, Marres H, Repping-Wuts H, Stikkelbroeck NM, Timmers HJ, Hermus AR, et al. High level of distress in long-term survivors of thyroid carcinoma: results of rapid screening using the distress thermometer. Acta Oncol 2013 Jan;52(1):128-137.

7. Lubitz CC, Kong CY, McMahon PM, Daniels GH, Chen Y, Economopoulos KP, Gazelle GS, Weinstein MC. Annual financial impact of well-differentiated thyroid cancer care in the United States. Cancer 2014 May;120(9):1345-1352.

8. Aschebrook-Kilfoy B, James B, Nagar S, Kaplan S, Seng V, Ahsan H, Angelos P, Kaplan EL, Guerrero MA, Kuo JH, et al. Risk factors for decreased quality of life in thyroid cancer survivors: initial findings from the North American Thyroid Cancer Survivorship Study. Thyroid 2015 Dec; 25(12):1313-1321.

9. Ramsey SD, Bansal A, Fedorenko CR, Blough DK, Overstreet KA, Shankaran V, Newcomb P. Financial insolvency as a risk factor for early mortality among patients with cancer. J Clin Oncol 2016 Mar;34(9):980-986.

10. Harari A, Li N, Yeh MW. Racial and socioeconomic disparities in presentation and outcomes of well-differentiated thyroid cancer. J Clin Endocrinol Metab 2014 Jan;99(1):133-141. 